\title{
HSV-1 ICP27 represses NF- $\kappa B$ activity by regulating Daxx sumoylation
}

\author{
Ji Ae Kim ${ }^{\#}$, Mi Sun Choi ${ }^{\dagger, \#}$, Jung Sun Min, Inho Kang, Jeongho Oh, Jin Chul Kim ${ }^{\ddagger}$ E Jeong Keun Ahn ${ }^{*}$ \\ Department of Microbiology \& Molecular Biology, College of Biological Science and Biotechnology, Chungnam National University, \\ Daejeon 34134, Korea
}

\begin{abstract}
Herpes simplex virus type 1 ICP27 is a multifunctional protein responsible for viral replication, late gene expression, and reactivation from latency. ICP27 interacts with various cellular proteins, including Daxx. However, the role of interaction between ICP27 and Daxx is largely unknown. Since Daxx is known to repress NF-kB activity, there is a possibility that ICP27 may influence the inhibitory effect of Daxx on NF-kB activity. In this study, we tested whether ICP27 affects the NF- $K B$ activity through its interaction with Daxx. Interestingly, ICP27 enhanced the Daxx-mediated repression of NF- $\mathrm{kB}$ activity. In addition, we found that sumoylation of Daxx regulates its interaction with p65. ICP27 binds to Daxx, inhibits Daxx sumoylation, and enhances $\mathrm{p} 65$ deacetylation induced by Daxx. Consequently, ICP27 represses the NF-KB activity, by elevating the inhibitory effect of Daxx on NF- $k B$ activity through desumoylation of Daxx. [BMB Reports 2017; 50(5): 275-280]
\end{abstract}

\section{INTRODUCTION}

Herpes simplex virus type 1 (HSV-1) is a neurotropic DNA virus that establishes latency within sensory neurons (1). During viral infection, the viral genes are expressed in a sequential cascade consisting of three major classes: immediate early $(\mathrm{IE})$, early $(\mathrm{E})$, and late $(\mathrm{L})$ genes. Transcription of the immediate early genes (ICP0, ICP4, ICP22, ICP27, and ICP47) is initiated by the cooperation between the host

*Corresponding author. Tel: +82-42-821-6418; Fax: +82-42-8227367; E-mail: jkahn@cnu.ac.kr

${ }^{\dagger}$ Current address: Department of predictive toxicology, Korea Institute of Toxicology (KIT), Daejeon 34114, Korea,

${ }^{\ddagger}$ Current address: Division of Biological Sciences, University of California, San Diego, La Jolla, CA, USA

${ }^{\#}$ These authors contributed equally to this work.

https://doi.org/10.5483/BMBRep.2017.50.5.010

Received 18 January 2017, Revised 1 February 2017, Accepted 16 March 2017

Keywords: Acetylation, Daxx, ICP27, NF-кB, Sumoylation transcription factor Oct-1 and VP16 protein, which is packed in the tegument of HSV-1 particle (2). These immediate early proteins serve as regulatory proteins that initiate the transcription of early genes, and are important in subsequent viral early protein expression (3).

Among the immediate early proteins of HSV-1, ICP27 is a multifunctional regulatory protein required for virus replication, and the switch from early to late viral gene expression (4). ICP27 also activates viral DNA replication and stimulates the transcription of late genes (5). In addition to gene expression regulation, ICP27 controls cell cycle and apoptosis through modulating signaling pathways such as NF- $\kappa B$ and MAPK pathways $(6,7)$. The activity of NF- $\kappa B$ is strongly augmented in macrophages infected with the ICP27-deletion mutant HSV-1, suggesting that ICP27 represses NF-אB (8).

Transcription factor NF- $\kappa B$ acts as a key regulator of cellular processes such as cell proliferation, survival, apoptosis, inflammation, and immune responses (9). A heterodimer consisting of two subunits p50 and p65 is the most abundant and ubiquitously expressed NF- $\kappa \mathrm{B}$, while the p65 subunit is the main transcriptional activator (10). In most unstimulated cells, the NF- $\mathrm{B} B$ proteins remain inactive through direct

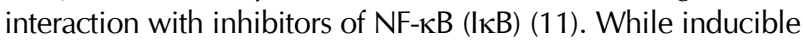
degradation of $\mathrm{I} \kappa \mathrm{B}$ proteins by various stimuli, including pro-inflammatory cytokines, $\mathrm{T}$ - and B-cell receptor signals, and viral and bacterial products, is a central mechanism regulating $\mathrm{NF}-\kappa \mathrm{B}$ transcriptional activity, recent studies indicate that posttranslational modification of NF- $\mathrm{KB}$ subunits, especially p65, is important to control the NF-kB activity (12). Studies indicate that the acetylation and phosphorylation of p65 plays a significant role in the regulation of NF- $\mathrm{kB}$ activity (13).

The NF- $\mathrm{BB}$ p65 subunit is reversibly acetylated at a number of sites, and this modification has variable effects on its transcriptional activity (14). Acetylation of p65 also modulates its DNA binding, subcellular localization, and assembly with IкBs. The transcriptional coactivators p300/CREB-binding protein (CBP) and p300/CBP-associated factor (PCAF) acetylate p65, while histone deacetylase 3 (HDAC3) deacetylates p65 (15). The p300/CBP mediated acetylation of p65 is especially inhibited by Daxx (16).

Daxx was initially identified as a protein that specifically binds to the death domain of Fas receptor, and enhances the

ISSN: 1976-670X (electronic edition)

Copyright (C) 2017 by the The Korean Society for Biochemistry and Molecular Biology

c) This is an open-access article distributed under the terms of the Creative Commons Attribution Non-Commercial License (http://creativecommons.org/licenses/by-nc/4.0) which permits unrestricted non-commercial use, distribution, and reproduction in any medium, provided the original work is properly cited. 
Fas-mediated apoptosis via JNK activation (17). In addition to apoptosis, Daxx represses several transcription factors, including ETS, Pax3, p53, Smad4, and NF-kB (18). Furthermore, Daxx interacts with ICP4 and ICP27, resulting in the nuclear domain 10 (ND10) deposition of HSV-1 viral genome (19).

We previously demonstrated that in HSV-1 infection, the $\mathrm{NF}-\mathrm{\kappa B}$ activity is repressed by ICP27 through stabilization of $I \kappa B \alpha$, whereas Daxx represses the NF- $\kappa B$ transcriptional activity by inhibiting NF- $\kappa B$ acetylation $(16,20)$. Based on these findings, we hypothesized that repression of NF- $\mathrm{kB}$ may be through interaction of ICP27 with Daxx. This study ascertains that ICP27 inhibits p65 acetylation and NF-KB transcriptional activity by repressing Daxx sumoylation, which results in an increased interaction between p65 and Daxx.

A

B

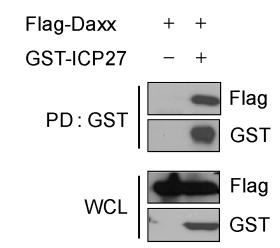

C
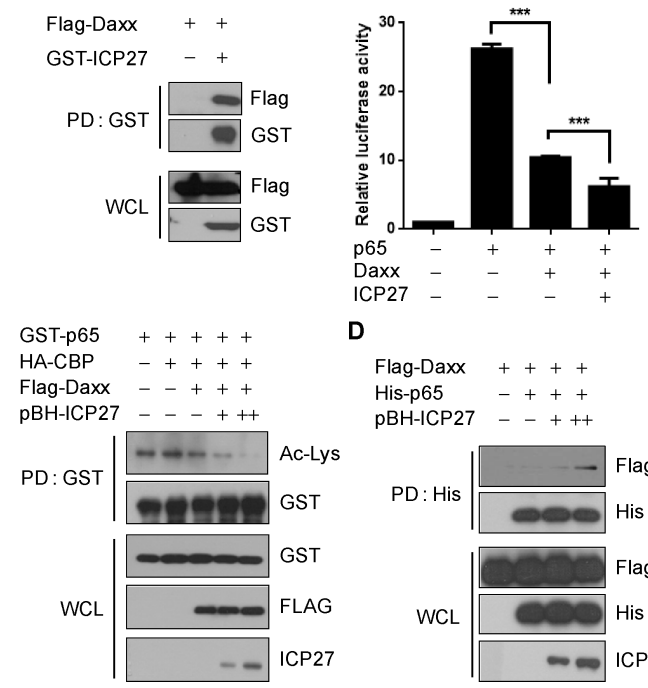

D

Flag-Daxx ++++ His-p65 -+++ pBH-ICP27 - - + ++

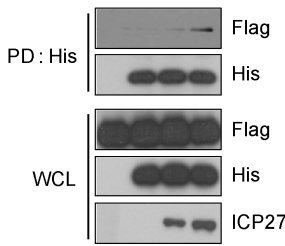

Fig. 1. ICP27 represses NF- $\kappa B$ activity by enhancing p65 deacetylation through its direct interaction with Daxx. (A) 293T cells were transfected with plasmids expressing Flag-ICP27 and GST-Daxx. GST-Daxx (3 $\mu$ g each) was pulled-down with glutathione-Sepharose $4 \mathrm{~B}$, and the protein complex was analyzed by immunoblotting using anti-Flag antibody. (B) 293T cells were transfected with NF-кB-luciferase reporter plasmid $(0.4 \mu \mathrm{g})$ and expression plasmids for p65 $(0.2 \mu \mathrm{g})$, $\operatorname{Daxx}(0.2 \mu \mathrm{g})$, and ICP27 plasmid $(0.2 \mu \mathrm{g})$. At $24 \mathrm{~h}$ post transfection, NF-kB activities were determined by a Dual-luciferase reporter assay system. Data represent three independent experiments performed in duplicate. Statistical significance was evaluated using Student's $t$ test $(* * * P<0.001)$. (C) 293T cells were transfected with combinations of plasmids expressing GST-p65, Flag-Daxx, HA-CBP, and ICP27 (1.5 $\mu$ g each). At $24 \mathrm{~h}$ after transfection, cells were treated with 2 um TSA for $30 \mathrm{~min}$. Cell extracts were pulled down with glutathione-Sepharose 4B, and analyzed by SDS-PAGE. The level of p65 acetylation was detected by immunoblotting using anti-acetylated lysine antibody. (D) 293T cells were transfected with plasmids expressing Flag-Daxx, His-p65 and ICP27 (2 $\mu \mathrm{g}$ each). At $24 \mathrm{~h}$ after transfection, cell extracts were pulled down with Ni-NTA Chelating Agarose CL-6B. Protein complex was separated by SDS PAGE and analyzed by Western blotting with specific antibodies.

\section{RESULTS}

ICP27 represses NF-kB activity by enhancing p65 deacetylation through its direct interaction with Daxx It has previously been reported that ICP27, ICP4, and Daxx possibly form a complex at ND10 in HSV-1 infected cells (19). Our investigation of the physical interaction between ICP27 and Daxx by the pull-down assay (Fig. 1A) revealed that ICP27 directly binds to Daxx. Since ICP27 inhibits NF- $\kappa B$ activity by regulation of $I \kappa B \alpha(16)$, and Daxx suppresses the acetylation of p65 (20), we assume that ICP27 might also be involved in the regulation of $\mathrm{NF}-\mathrm{\kappa B}$ through Daxx. We therefore examined whether ICP27 was involved in regulating NF- $\mathrm{KB}$ activity affected by Daxx. As expected, Daxx represses the NF-kB activity, and ICP27 further suppresses this repressed NF-אB activity (Fig. 1B).

Since Daxx inhibits p65 acetylation (16), we examined whether this acetylation is affected by the interaction between ICP27 and Daxx. To assess the effect of ICP27 on p65 acetylation regulated by Daxx, 293T cells were cotransfected with expression plasmids for p65, CBP, Daxx, and ICP27, following which they were treated with TSA, a HDACs inhibitor, to diminish the artifacts by HDACs. Daxx-mediated inhibition of p65 acetylation is not affected by TSA (16), while HDACs are capable of deacetylating p65 (14). As expected, Daxxx decreases the level of p65 acetylation, which is further reduced dramatically by ICP27 in a dose dependent manner (Fig. 1C). To exclude the possibility that ICP27 directly inhibits p65 acetylation, we evaluated the physical interaction between ICP27 and p65 by the pull-down assay, and identified that ICP27 does not directly bind to p65 (data not shown). Because ICP27 does not physically interact with p65, we further examined whether ICP27 affects the binding between

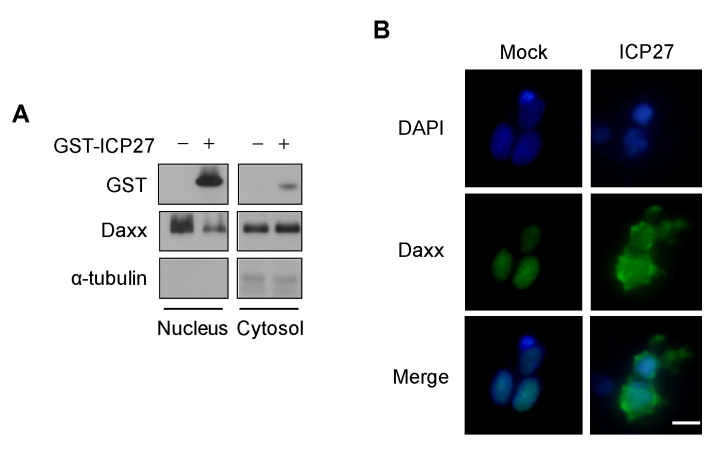

Fig. 2. ICP27 changes subcellular distribution of Daxx. (A) 293T cells were transfected with plasmid for GST- ICP27 $(6 \mu \mathrm{g})$. At 24 $\mathrm{h}$ after transfection, cells were fractionated into nuclear and cytoplasmic fraction. Fractionated proteins were immunoblotted with anti-GST and anti-Daxx antibodies. Tubulin was used as a cytosolic marker. (B) SH-SY5Y cells were transfected with plasmid for GSTICP27 (3 $\mu$ g). At $24 \mathrm{~h}$ after transfection, subcellular distribution of Daxx (green) was determined by immunofluorescence microscopy, using specific antibody against Daxx. Scale bar $=5 \mu \mathrm{m}$. 
Daxx and p65 to regulate p65 acetylation. As seen in Fig. 1D, ICP27 augments the interaction between Daxx and p65. Taken together, these data suggest that ICP27 affects p65 acetylation by enhancing the interaction between Daxx and p65.

\section{ICP27 changes cellular localization of Daxx}

To further understand how ICP27 enhances the interaction between Daxx and p65, we next examined whether ICP27 affects the subcellular localization of Daxx. While ICP27 shuttles between the nucleus and the cytoplasm, Daxx mainly exists in the nucleus and p65 is predominantly localized in the cytoplasm at normal state. We speculated that ICP27 might be altering the cellular localization of Daxx. A subcellular fractionation assay was performed to analyze the intracellular distribution of Daxx in the presence of ICP27. Interestingly,

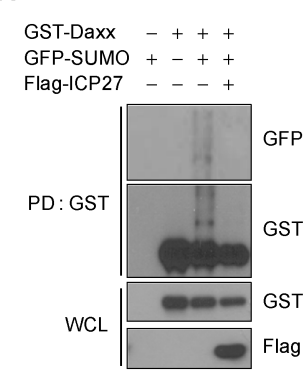

C

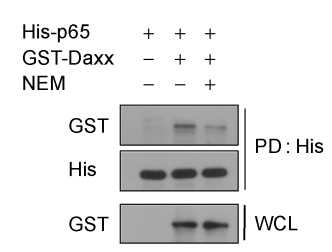

B

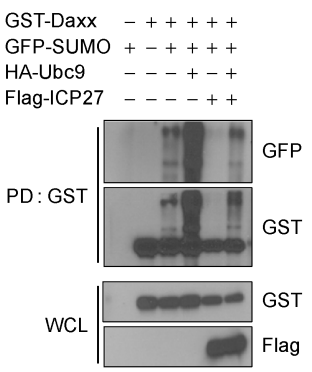

D

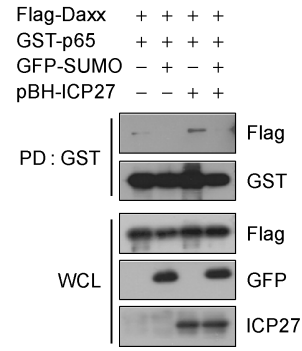

Fig. 3. ICP27 increases the interaction between Daxx and p65 by regulating Daxx sumoylation. (A) 293T cells were transfected with plasmids expressing GST-Daxx, GFP-SUMO-1, and Flag-ICP27 (2 $\mu \mathrm{g}$ each). At $24 \mathrm{~h}$ after transfection, cell extracts were prepared with lysis buffer containing $20 \mathrm{mM}$ NEM and pulled down with glutathione-Sepharose 4B. Sumoylated Daxx was detected by immunoblotting using anti-GFP and anti-GST antibodies. (B) 293T cells were transfected with plasmids expressing GST-Daxx, GFPSUMO-1, HA-Ubc9, and Flag-ICP27 (1.5 $\mu \mathrm{g}$ each). At $24 \mathrm{~h}$ after transfection, cell extracts containing $20 \mathrm{mM}$ NEM were pulled down with glutathione-Sepharose 4B, and analyzed by Western blotting. (C) 293T cells were transfected with plasmids expressing Flag-Daxx and His-p65 (3 $\mu \mathrm{g}$ each). At $24 \mathrm{~h}$ after transfection, cell extracts containing NEM were pulled down with Ni-NTA Chelating Agarose CL-6B, and analyzed by immunoblotting using anti-Flag antibody. (D) 293T cells were transfected with plasmids expressing Flag-Daxx, GST-p65, GFP-SUMO, and ICP27 (1.5 $\mu \mathrm{g}$ each). At $24 \mathrm{~h}$ after transfection, cell extracts were pulled down and analyzed by Western blotting with specific antibodies.
ICP27 elevated the translocation of Daxx from the nucleus to the cytoplasm (Fig. 2A). Immunofluorescence analysis also demonstrated that Daxx is relocated to the cytosol in the presence of ICP27 (Fig. 2B). These data suggest that ICP27 increases co-localization of Daxx and p65 in the cytoplasm.

\section{ICP27 inhibits Daxx sumoylation}

Recent studies show that Daxx sumoylation is important for Daxx localization. Sumoylation-defective Daxx mutants localize in the cytoplasm, whereas wild type Daxx localize in the nucleus (21). Because ICP27 changes the subcellular localization of Daxx, further examination of the regulation revealed that ICP27 inhibited Daxx sumoylation (Fig. 3A), and Daxx sumoylation induced by SUMO E2 conjugation enzyme Ubc9 is also inhibited by ICP27 (Fig. 3B). To further substantiate the effect of Daxx sumoylation on the interaction between Daxx and p65, we performed the pull-down assay using lysis buffer containing NEM, which is commonly used as a desumoylation inhibitor. As shown in Fig. 3C, NEM abolishes the physical binding of Daxx to p65, thereby suggesting that the elevated sumoylation of Daxx interferes the interaction between Daxx and p65. Moreover, the inhibition of Daxx sumoylation by ICP27 increases the interaction between Daxx and p65 (Fig. 3D). These results suggest that the sumoylation level of Daxx is critical for the interaction between Daxx and p65.

\section{In HSV-1 infection, ICP27 represses p65 acetylation by inhibiting Daxx sumoylation}

Next, we investigated whether ICP27 influences p65 acetylation during early HSV-1 infection. 293T cells were infected with HSV-1 wild type virus (wt) and ICP27 deletion mutant

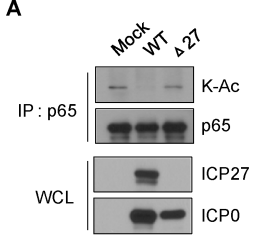

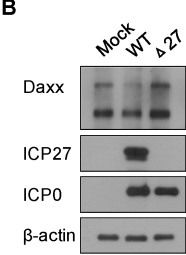

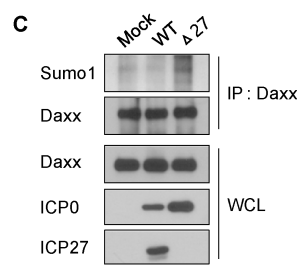

Fig. 4. ICP27 represses p65 acetylation through interaction with Daxx at early stage of HSV-1 infection. (A) 293T cells were infected with wild type HSV-1 and HSV-1 $\Delta 27$ virus at MOI of 5. At $2 \mathrm{~h}$ post infection, cells were treated with $1 \mu \mathrm{m}$ TSA for 4 h. At $6 \mathrm{~h}$ post infection, cells were harvested in modified RIPA buffer containing $1 \mu \mathrm{m}$ TSA and precipitated with anti-p65 antibody. The immunocomplex was analyzed by Western blotting with the indicated antibodies. (B) 293T cells were infected with wild type HSV-1 and HSV-1 $\Delta 27$ virus at MOI of 5 . At $6 \mathrm{~h}$ post infection, cells were harvested in modified RIPA buffer with 20 $\mathrm{mM} N E M$, and analyzed by Western blotting with the indicated antibodies. (C) $293 \mathrm{~T}$ cells were infected with wild type HSV-1 and HSV-1 $\Delta 27$ virus at MOI of 5 . At $6 \mathrm{~h}$ post infection, cells were harvested in modified RIPA buffer with $20 \mathrm{mM} \mathrm{NEM}$ and precipitated with anti-Daxx antibody. The immunocomplex was analyzed by Western blotting, with the indicated antibodies. 
virus $(\Delta 27)$ at $\mathrm{MOI} 5$. We observed that p65 acetylation is repressed in HSV-1 wt infected cells as compared with mock infected cells, whereas p65 acetylation is elevated in HSV-1 $\Delta 27$ infected cells as compared with HSV-1 wt infected cells (Fig. 4A). We further examined whether HSV-1 infection affected Daxx sumoylation. At $6 \mathrm{~h}$ post infection, 293T cells were harvested with lysis buffer containing NEM to inhibit desumoylation of proteins. In HSV-1 $\Delta 27$ infected cells, Daxx sumoylation is more increased as compared with the HSV-1 wt infected cells (Fig. 4B and C), suggesting that ICP27 represses Daxx sumoylation with HSV-1 virus infection. Our studies thus reveal that ICP27 represses p65 acetylation by inhibiting Daxx sumoylation at early stages of HSV-1 infection.

\section{DISCUSSION}

NF-KB plays a crucial role in regulating cellular processes such as apoptosis, proliferation, inflammation, and immune responses $(22,23)$. Viruses challenge the host immune system, and have evolved different strategies to regulate NF-kB signaling (24). Some viruses modulate NF- $\mathrm{kB}$ bidirectionally in immune surveillance, optimization of viral infection, and viral replication (25). Especially in HSV-1 infection, diverse viral proteins maintain a delicate balance between activation and inhibition of NF-кB.

In general, HSV-1 activates NF-kB in a biphasic way. The first wave of NF- $\mathrm{BB}$ activation is mediated by glycoprotein $\mathrm{D}$ and viral tegument protein UL37, which bind to herpesvirus entry mediator (HVEM) (26) and TRAF6, respectively (27). In the second wave, the IE viral proteins ICP4 and ICP27, activate $\mathrm{NF}-\mathrm{KB}$ in IKK-dependent manner (28). However, recent studies reveal that HSV-1 IE proteins have a repressible role in NF-KB signaling. ICP0 inhibits TLR2-dependent activation of NF- $\mathrm{KB}$ signaling by degradation of the myeloid differentiation primary response (MYD88) (29). Also, early viral infection suppresses the NF- $\mathrm{kB}$ activity, while there is no suppression by the HSV-1 $\Delta 27$ virus. However, at late HSV-1 infection, HSV-1 wt and HSV-1 $\Delta 27$ both promote NF-kB activities (20). These results suggest that ICP27 plays a bifunctional role in NF- $\mathrm{KB}$ regulation. Thus, more precise studies on the molecular mechanism of NF- $\kappa \mathrm{B}$ modulation by ICP27 is required.

In this study, we provide experimental evidence of a novel mechanism for ICP27 to inhibit NF-kB activity, through regulation of Daxx sumoylation, suggesting that ICP27 physically interacts with Daxx to inhibit Daxx sumoylation, possibly by steric interference. Sumoylation is an important post-translational modification that regulates protein activity, stability, and subcellular localization. Also, Daxx itself is sumoylated by SUMO-1 and Ubc9 E2-conjugating enzyme (30). Several recent publications demonstrate that Daxx sumoylation is associated with its subcellular localization. Ubc9 dominant-negative mutant induces accumulation of Daxx to cytoplasm (31). Experiments using sumoylation-defective Daxx KA mutants revealed that the physiological role of Daxx sumoylation is regulating its nuclear anchoring (21). Our data also imply that Daxx sumoylation is crucial for its subcellular localization. ICP27 changes the cellular localization of Daxx from the nucleus to the cytoplasm through repression of Daxx sumoylation, and subsequently ICP27 increases the interaction between 065 and Daxx. In addition, Daxx plays a role as a regulatory protein in viral gene expressions and intrinsic immunity against viral infection. Many viruses modulate Daxx for viral replications and immune escapes in host cells (32).

We have previously reported that Daxx inhibits p65 acetylation (16). NF- $\kappa B$ transcriptional activity is regulated by diverse posttranslational modifications, including phosphorylation, methylation, ubiquitination, and acetylation. Seven acetylated lysines have been identified within p65; acetylation of K221 and K310 are especially important for NF-KB transcriptional activity. Acetylation of K221 enhances the NF- $\mathrm{KB}$ DNA binding affinity and reduces interaction with $1 \kappa B \alpha$. Acetylation of $\mathrm{K} 310$ is required for full NF- $\mathrm{KB}$ activity, but does not affect either NF-kB DNA binding affinity or its assembly with I $\mathrm{kB} \alpha$ (33). Daxx binds to the $\mathrm{N}$-terminal domain of p65, including major acetylation sites, and it appears that Daxx interferes with p65 acetylation by steric hindrance (16). In this report, we demonstrated that ICP27 inhibits p65 acetylation through increasing the interaction between Daxx and p65. Owing to this increased interaction induced by ICP27 through Daxx desumoylation, ICP27 represses NF-kB transcriptional activity. This is consistent with our previous report that NF- $\mathrm{kB}$ transcriptional activity is more repressed at the early phase of viral infection in HSV-1 wt infected cells, compared with in HSV-1 $\Delta 27$ infected cells (20). Because activation of NF- $\mathrm{KB}$ is an essential step of immune response activation, many viruses disrupt the NF- $\kappa B$ pathway. Therefore, we conclude here a possible mechanism, that HSV-1 is able to escape immune surveillance in infected cells through modulating the NF- $\mathrm{KB}$ activity by regulation of Daxx sumoylation.

\section{MATERIALS AND METHODS}

\section{Cells and Reagents}

HEK 293T, Vero, 3-3 (HSV-1 ICP27 expressing Vero), and SH-SY5Y cells were maintained in Dulbecco's modified Eagle's medium (DMEM) supplemented with $10 \%$ fetal bovine serum (FBS), at $37^{\circ} \mathrm{C}$ in $5 \% \mathrm{CO}_{2}$. 3-3 cells were kind gifts from Dr. P. Schaffer (Harvard University, USA). Cells were transfected using PEI (Sigma-Aldrich) according to the manufacturer's instruction. Trichostatin A (TSA) and N-ethylmaleimide (NEM) were purchased from Sigma-Aldrich.

\section{Viruses and virus infection}

HSV-1 wild type strain KOS, and ICP27 deletion mutant virus (34), were propagated and titrated on Vero cells and 3-3 cells, respectively. For virus infection, cell monolayers were infected with either KOS or mutant virus at a multiplicity of infection (MOI) of 5 plaque forming units (PFU)/cell. 


\section{Plasmids}

ICP27, Daxx, p65, and CBP expression plasmids have been described previously (20). pBH-ICP27 was provided by Dr. S. Rice (35). Plasmids expressing EGFP-SUMO-1 and HA-UBC9 were provided by Dr. H. Shih (36) and Dr. H. Ariga (37), respectively.

\section{GST and Ni-NTA Pull-down assay}

Transiently transfected 293T cells were lysed in modified RIPA buffer $(50 \mathrm{mM}$ Tris-HCl pH 7.4, 1\% NP-40, 0.25\% Nadeoxycholate, $150 \mathrm{mM} \mathrm{NaCl}, 1 \mathrm{mM}$ EDTA, $1 \mathrm{mM}$ PMSF, $1 \mathrm{mM}$ $\mathrm{NaF}, 1 \mathrm{mM} \mathrm{Na} \mathrm{VO}_{4}, 1 \mu \mathrm{g} / \mathrm{ml}$ aprotinin, $1 \mu \mathrm{g} / \mathrm{ml}$ leupeptin, and $1 \mu \mathrm{g} / \mathrm{ml}$ pepstatin) for $30 \mathrm{~min}$ at $4^{\circ} \mathrm{C}$. Cell lysates were incubated overnight at $4^{\circ} \mathrm{C}$, with glutathione-Sepharose $4 \mathrm{~B}$ beads or Ni-NTA beads (Incospharm). The bead complexes were washed three times with modified RIPA buffer, and bound proteins were analyzed by Western blotting.

\section{Immunoprecipitation and Western Blot Analysis}

Cell lysates were prepared in modified RIPA buffer and incubated with specific antibody overnight at $4^{\circ} \mathrm{C}$. The immunoprecipitated complexes were isolated using protein-A Sepharose, resolved by SDS-PAGE, and transferred to nitrocellulose membrane. Immunoblotting was performed with various antibodies. Anti-GST, anti-His, anti-HA, anti-GFP, anti-ICP27, rabbit anti-goat IgG-HRP, and goat anti-mouse IgG-HRP antibodies were purchased from Santa Cruz. Anti-Flag antibody was purchased from Sigma-Aldrich.

\section{Luciferase assay}

All luciferase assays were performed at $24 \mathrm{~h}$ after transfection, using a Dual Luciferase Assay kit (Promega) according to the manufacturer's instruction. 293T cells were transiently transfected with luciferase reporter construct NF-KB-Luc and pTK Renilla luciferase plasmid (Promega) using PEI, according to the manufacturer's instruction.

\section{Subcellular fractionation assay}

Briefly, cells were suspended in fractionation buffer $(250 \mathrm{mM}$ sucrose, $20 \mathrm{mM}$ HEPES $\mathrm{pH} 7.4,10 \mathrm{mM} \mathrm{KCl}, 1.5 \mathrm{mM} \mathrm{MgCl}_{2}, 1$ mM EDTA, $1 \mathrm{mM}$ EGTA, $1 \mathrm{mM}$ DTT, $1 \mathrm{mM}$ PMSF, $1 \mathrm{mM} \mathrm{NaF}$, $1 \mathrm{mM} \mathrm{Na}{ }_{3} \mathrm{VO}_{4}, 1 \mu \mathrm{g} / \mathrm{ml}$ aprotinin, $1 \mu \mathrm{g} / \mathrm{ml}$ leupeptin, and 1 $\mu \mathrm{g} / \mathrm{ml}$ pepstatin). Cell lysate was passed 10 times through a 25 Ga needle using a $1 \mathrm{ml}$ syringe, and stored on ice for $20 \mathrm{~min}$. The lysates were centrifuged at 3,000 rpm for $5 \mathrm{~min}$ at $4^{\circ} \mathrm{C}$, and supernatants were collected as cytosolic fractions. The nuclear pellets were resuspended in nuclear buffer (RIPA buffer with $10 \%$ glycerol and $0.1 \%$ SDS) and sonicated in three $10 \mathrm{sec}$ bursts at $40 \%$ intensity, with $30 \mathrm{sec}$ break on ice.

\section{Immunofluorescence assay}

Transfected cells on sterile glass coverslips were fixed for 10 min with $4 \%$ paraformaldehyde at room temperature, and permeabilized with PBS containing $0.2 \%$ Triton X-100 for 5 min. Cells were immunostained with primary antibody (antiDaxx, Santa Cruz), overnight at $4^{\circ} \mathrm{C}$, and then treated with fluorescent secondary antibody (donkey anti-rabbit Alexa Fluor 488, Invitrogen) for $1 \mathrm{~h}$ at room temperature. Fluorescence microscopy was performed using a Leica TCS SL system.

\section{Statistical analysis}

Statistical comparisons of results were evaluated by one-way analysis of variance (ANOVA) followed by Student's $t$ test for unpaired values. A P value of less than 0.001 was considered significant.

\section{ACKNOWLEDGEMENTS}

This work was supported by the research fund of Chungnam National University.

\section{CONFLICTS OF INTEREST}

The authors have no conflicting financial interests.

\section{REFERENCES}

1. Cook ML and Stevens JG (1973) Pathogenesis of herpetic neuritis and ganglionitis in mice: evidence for intra-axonal transport of infection. Infect Immun 7, 272-288

2. O'Hare P and Goding CR (1988) Herpes simplex virus regulatory elements and the immunoglobulin octamer domain bind a common factor and are both targets for virion transactivation. Cell 52, 435-445

3. Honess RW and Roizman B (1974) Regulation of herpesvirus macromolecular synthesis. I. Cascade regulation of the synthesis of three groups of viral proteins. J Virol 14, 8-19

4. Sacks WR, Greene CC, Aschman DP and Schaffer PA (1985) Herpes simplex virus type 1 ICP27 is an essential regulatory protein. J Virol 55, 796-805

5. McCarthy AM, McMahan L and Schaffer PA (1989) Herpes simplex virus type 1 ICP27 deletion mutants exhibit altered patterns of transcription and are DNA deficient. J Virol 63, 18-27

6. Hargett D, Rice S and Bachenheimer SL (2006) Herpes simplex virus type 1 ICP27-dependent activation of NFkappaB. J Virol 80, 10565-10578

7. Gillis PA, Okagaki LH and Rice SA (2009) Herpes simplex virus type 1 ICP27 induces p38 mitogen-activated protein kinase signaling and apoptosis in HeLa cells. J Virol 83, 1767-1777

8. Melchjorsen J, Siren J, Julkunen I, Paludan SR and Matikainen S (2006) Induction of cytokine expression by herpes simplex virus in human monocyte-derived macrophages and dendritic cells is dependent on virus replication and is counteracted by ICP27 targeting NF-kappaB and IRF-3. J Gen Virol 87, 1099-1108

9. Hayden MS and Ghosh S (2004) Signaling to NF-kappaB. Genes Dev 18, 2195-2224

10. Ballard DW, Dixon EP, Peffer NJ et al (1992) The 65-kDa 
subunit of human NF-kappa B functions as a potent transcriptional activator and a target for v-Rel-mediated repression. Proc Natl Acad Sci U S A 89, 1875-1879

11. Ghosh S, May MJ and Kopp EB (1998) NF-kappa B and Rel proteins: evolutionarily conserved mediators of immune responses. Annu Rev Immunol 16, 225-260

12. Huang B, Yang XD, Lamb A and Chen LF (2010) Posttranslational modifications of NF-kappaB: another layer of regulation for NF-kappaB signaling pathway. Cell Signal 22, 1282-1290

13. Perkins ND (2006) Post-translational modifications regulating the activity and function of the nuclear factor kappa B pathway. Oncogene 25, 6717-6730

14. Kiernan R, Bres V, Ng RW et al (2003) Post-activation turn-off of NF-kappa B-dependent transcription is regulated by acetylation of p65. J Biol Chem 278, 2758-2766

15. Chen L, Fischle W, Verdin E and Greene WC (2001) Duration of nuclear NF-kappaB action regulated by reversible acetylation. Science 293, 1653-1657

16. Park J, Lee JH, La M et al (2007) Inhibition of NF-kappaB acetylation and its transcriptional activity by Daxx. J Mol Biol 368, 388-397

17. Yang $X$, Khosravi-Far R, Chang HY and Baltimore D (1997) Daxx, a novel Fas-binding protein that activates JNK and apoptosis. Cell 89, 1067-1076

18. Salomoni P and Khelifi AF (2006) Daxx: death or survival protein? Trends Cell Biol 16, 97-104

19. Tang Q, Li L, Ishov AM, Revol V, Epstein AL and Maul GG (2003) Determination of minimum herpes simplex virus type 1 components necessary to localize transcriptionally active DNA to ND10. J Virol 77, 5821-5828

20. Kim JC, Lee SY, Kim SY et al (2008) HSV-1 ICP27 suppresses NF-kappaB activity by stabilizing IkappaBalpha. FEBS Lett 582, 2371-2376

21. Muromoto R, Ishida M, Sugiyama K et al (2006) Sumoylation of Daxx regulates IFN-induced growth suppression of B lymphocytes and the hormone receptor-mediated transactivation. J Immunol 177, 1160-1170

22. Karin M, Cao YX, Greten FR and Li ZW (2002) NF-kappa $B$ in cancer: From innocent bystander to major culprit. Nat Rev Cancer 2, 301-310

23. Li QT and Verma IM (2002) NF-kappa B regulation in the immune system. Nat Rev Immunol 2, 725-734

24. Kim SY, Kim JC, Kim JK et al (2008) Hepatitis B virus X protein enhances NFkappaB activity through cooperating with VBP1. BMB Rep 41, 158-163

25. Santoro MG, Rossi A and Amici C (2003) New embo member's review - NF-kappa B and virus infection: who controls whom. EMBO J 22, 2552-2560

26. Sciortino MT, Medici MA, Marino-Merlo F et al (2008) Involvement of HVEM receptor in activation of nuclear factor kappa B by herpes simplex virus 1 glycoprotein D. Cell Microbiol 10, 2297-2311

27. Liu $X Q$, Fitzgerald $K$, Kurt-Jones E, Finberg $R$ and Knipe DM (2008) Herpesvirus tegument protein activates NFkappa B signaling through the TRAF6 adaptor protein. Proc Natl Acad Sci U S A 105, 11335-11339

28. Hargett D, Rice S and Bachenheimer SL (2006) Herpes simplex virus type IICP27-Dependent activation of NFkappa B. J Virol 80, 10565-10578

29. van Lint AL, Murawski MR, Goodbody RE et al (2010) Herpes Simplex Virus Immediate-Early ICPO Protein Inhibits Toll-Like Receptor 2-Dependent Inflammatory Responses and NF-kappa B Signaling. J Virol 84, 10802-10811

30. Jang MS, Ryu SW and Kim E (2002) Modification of Daxx by small ubiquitin-related modifier-1. Biochem Biophys Res Commun 295, 495-500

31. Mo YY, Yu Y, Ee PL and Beck WT (2004) Overexpression of a dominant-negative mutant Ubc9 is associated with increased sensitivity to anticancer drugs. Cancer Res 64, 2793-2798

32. Schreiner S and Wodrich H (2013) Virion Factors That Target Daxx To Overcome Intrinsic Immunity. J Virol 87, 10412-10422

33. Chen LF, Mu Y and Greene WC (2002) Acetylation of RelA at discrete sites regulates distinct nuclear functions of NF-kappaB. EMBO J 21, 6539-6548

34. McMahan L and Schaffer PA (1990) The repressing and enhancing functions of the herpes simplex virus regulatory protein ICP27 map to C-terminal regions and are required to modulate viral gene expression very early in infection. J Virol 64, 3471-3485

35. Rice SA and Knipe DM (1988) Gene-Specific Transactivation by Herpes-Simplex Virus Type-1 Alpha-ProteinIcp27. J Virol 62, 3814-3823

36. Kuo HY, Chang CC, Jeng JC et al (2005) SUMO modification negatively modulates the transcriptional activity of CREB-binding protein via the recruitment of Daxx. Proc Natl Acad Sci U S A 102, 16973-16978

37. Shinbo Y, Niki T, Taira T et al (2006) Proper SUMO-1 conjugation is essential to DJ-1 to exert its full activities. Cell Death Differ 13, 96-108 